\title{
APPLICATIONS OF 3D-EDGE DETECTION FOR ALS POINT CLOUD
}

\author{
H. Ni ${ }^{\text {a, }}$, X. G. Lin ${ }^{\text {a }}$ J. X. Zhang ${ }^{b}$ \\ ${ }^{a}$ Institute of Photogrammetry and Remote Sensing, Chinese Academy of Surveying and Mapping, No. 28, Lianhuachixi Road, \\ Haidian District, Beijing 100830, China - nih2015@yeah.net, linxiangguo@gmail.com \\ b National Quality Inspection and Testing Center for Surveying and Mapping Products, No. 28, Lianhuachixi Road, Haidian District, \\ Beijing 100830, China - zhangjx@casm.ac.cn
}

Commission VI, WG VI/4

KEY WORDS: 3D-Edge, Straight Line Segment, Filtering, Airborne Laser Scanning Point Cloud, Random Sample Consensus, Ground Breaklines

\begin{abstract}
:
Edge detection has been one of the major issues in the field of remote sensing and photogrammetry. With the fast development of sensor technology of laser scanning system, dense point clouds have become increasingly common. Precious 3D-edges are able to be detected from these point clouds and a great deal of edge or feature line extraction methods have been proposed. Among these methods, an easy-to-use 3D-edge detection method, AGPN (Analyzing Geometric Properties of Neighborhoods), has been proposed. The AGPN method detects edges based on the analysis of geometric properties of a query point's neighbourhood. The AGPN method detects two kinds of 3D-edges, including boundary elements and fold edges, and it has many applications. This paper presents three applications of AGPN, i.e., 3D line segment extraction, ground points filtering, and ground breakline extraction. Experiments show that the utilization of AGPN method gives a straightforward solution to these applications.
\end{abstract}

\section{INTRODUCTION}

Benefiting from the advances in sensor technology for both airborne and ground-based mobile laser scanning, dense points clouds have become increasingly common, and the need for new approaches to address these point clouds has become increasingly important (Lin et al., 2015). In contrast to the $2 \mathrm{D}$ remote sensing imagery, a laser scanning point cloud is a swarm of points with XYZ coordinates (Zhang and Lin, 2017), and thus describes the 3D topographic profiles of natural surfaces. Therefore, laser scanning point clouds provide more geometric information than 2D images. However, it is a double-edged sword, which brings difficulties to extract low-level features. Among these low-level features, edges play an important role in a series of applications. To extract edges from laser scanning point clouds, 2D image processing methods cannot be applied directly due to the complex storage structure and scattered spatial neighborhood of $3 \mathrm{D}$ point clouds.

According to the aspect of process, current 3D edge detection methods can be divided into two categories. The first category is called "indirect method", it first converts 3D point cloud into image or registers 3D-point cloud and image, then, 2D edges are detected in image utilizing image processing, finally, 2D edges are back-projected into 3D-point cloud as 3D edges ( $\mathrm{Li}$ et al. 2013; Poullis 2013). But, when the "indirect methods" convert $3 \mathrm{D}$ point cloud into image, some spatial geometric information may be lost. The second category is called "direct method", it extracts 3D edges from 3D-point cloud directly (Sampath et al. 2010; Demarsin et al. 2007; Borges et al. 2010; Truong-Honget al. 2013). However, the "direct methods" must utilize the processes of point cloud segmentation or object recognition as supplements, their procedures are complex. Moreover, the most serious shortcoming of "direct methods" is that they cannot detect all kinds of edges in 3D point clouds.

To address the problems of direct methods, Ni et al. (2016) proposed an easy-to-use 3D edge detection method which is named AGPN (Analyzing Geometric Properties of Neighborhoods). It includes two main steps: edge detection and feature line tracing. In the edge detection step, AGPN first analyzes geometric properties of each query point's neighborhood, and then combines RANSAC (RANdom SAmple Consensus) and angular gap metric (Gumhold et al., 2001) to detect edges. In the feature line tracing step, feature lines are traced by a hybrid method based on region growing and model fitting in the detected edges (Ni et al., 2016). Moreover, this study defines 3D-edges as "3D discontinuities of the geometric properties in the underlying 3D-scene". AGPN method is able to extract two kinds of edges, i.e., boundary elements and fold edges, which include almost all types of edges in a laser scanning point cloud. AGPN method is able to be applied to a number of airborne laser scanning (ALS) point cloud applications, such as DLG (Digital Line Graphic) generation, building reconstruction, etc. In this paper, we focus on three applications, i.e., straight line segment extraction, ALS point cloud filtering, and ground breakline extraction.

To extract straight line segments from ALS point clouds, there are still two groups of methods, i.e., indirect methods, and direct methods. The procedure of indirect methods for extracting straight line segments is similar to that for 3D-edge detection. For this kind of methods, the study (Lin et al., 2015) is a typical approach. It project a $3 \mathrm{D}$ point cloud into multi-view images, and then employs a new 2D line segment detector LSD (Von Gioi, 2010) to extract lines in $2 \mathrm{D}$ images, which forwards by a backprojection procedure. This method (Lin et al., 2015) is robust and 
high-efficiency, however obtains 3D line segments in 2D space and the abundant geometric information of $3 \mathrm{D}$ point clouds is wasted. Direct methods mainly aim at the extraction of plane intersection lines (Borges et al, 2010) or break lines (Sampath and Shan, 2010). These methods first segment a laser scanning point cloud into planes, and then detect the relationship between different planes. If there are two adjacent planes, these studies intersect them and compute the plane intersection lines based on plane model coefficients. However, there are two drawbacks: (1) the task to fit a small and narrow plane is difficult, and (2) when the data become complex, these methods may generate unexpected lines at non-planar surfaces (Lin et al., 2015).

High-resolution DTMs (Digital Terrain Models) are essential for many geographic information system (GIS)-related analysis and visualization (Zhang et al., 2003). Ground surfaces derived from ALS point clouds meet this need, and a great deal of studies focus on it which are so called filtering. Among the existing ALS point cloud filtering methods, Axelsson's method (Axelsson, 1999) which utilizes TIN to densify the initial ground surface, makes an excellent performance, and it has been integrated into the commercial software TerraSolid. A group of filtering algorithm (Sohn and Dowman, 2002, Zhang and Lin, 2013, Lin and Zhang, 2014) which are called progressive-densification-based filters are derived from it. However, discontinuities in the bare ground pose great challenges to Axelsson's method (Zhang and Lin, 2013). To solve this problem, point cloud segmentation has been integrated into this method. In this case, constructing TIN using points in the initial ground surface is redundancy, therefore, Lin's method (Lin et al., 2016) utilize feature points of each surface segment to construct TIN. If smoothness segmentation (Zhang and Lin, 2013) is utilized, the surface depicted by a segment may be rugged and full of short break lines. Only utilizing boundaries to depict a surface segment is unreasonable.

To generate high quality DEMs (Digital Elevation Models), breaklines should also be considered as constraints in interpolating the grid DEMs or fixed edges in the TINs (Yang et al., 2016). Breaklines are classified into three types, i.e., jump breaklines, crease breaklines, and curvature breaklines (Brugelmann, 2000). To extract breaklines, ground points should be first extracted, then ground breaklines are generated from these ground points. Currently, there are few studies paying attention on ground breakline extraction. For example, Yang et al. (2016) presents a feasible way based on range images. The method converts point clouds into range images, and the abundant geometric information in point clouds is wasted. The 3D-edges which include boundary elements and fold edges detected by AGPN method make the direct way possible and may solve this problem.

According to the aforementioned review of the existing methods for ALS point cloud processing, AGPN has the capacity to improve the procedures for straight line segment extraction, ALS point cloud filtering, and ground breakline extraction. In the following sections, we will present how to improve these existing methods utilizing the 3D-edges detected by AGPN. We first present an overview of the AGPN method in Section 2. Next, the design of improving these existing methods is shown in Section 3 , and then, experimental validation of these improvements are shown in Section 4. At last, we give a conclusion in Section 5.

\section{A REVIEW OF THE AGPN METHOD}

There are two steps in AGPN, i.e., 3D-edge detection and feature line tracing, which are able to be found in Ni et al., (2016). The procedure of feature line tracing is briefly reviewed herein, because there are some changes in the feature line tracing when we improve the application of straight line segment extraction.

The feature line tracing of AGPN is a region-growing-based method. The key step of feature line tracing is the neighbourhood refinement. The method first obtains the nearest neighbors of a query point by using the $k d$-tree algorithm. Next, a straight line model is fitted by the RANSAC algorithm, and then, the nearest neighbors are divided into inliers and outliers. The inliers containing the query point are the refined nearest neighbors. Otherwise, the outliers are processed iteratively by the RANSAC algorithm until the updated inliers contain the query point.

The feature line tracing procedure is composed of the following steps:

a. Select a query point as the initial seed in the extracted edges. Then, the initial seed is added to a point queue. If all the edge points are traversed, go to the end.

b. Select a point in the current point queue as the current seed. The refined neighborhood of the current seed is determined.

c. Compute the principle direction of the current seed based on its refined neighborhood, and compute each neighbor's principle direction in the same way. If a neighbor's principle direction is similar to that of the current seed, the neighbor will be added into the current point queue.

d. Iterative the steps b-c until all the points in the current point queue are traversed.

e. Iterative the steps a-d until all the points in the extracted edges are traversed.

Results of the AGPN method are shown in figures 1-2. From the result, we can find that all the $3 \mathrm{D}$ edges are detected (see figure 1 ), and feature lines in the extracted edges are traced (see figure 2).
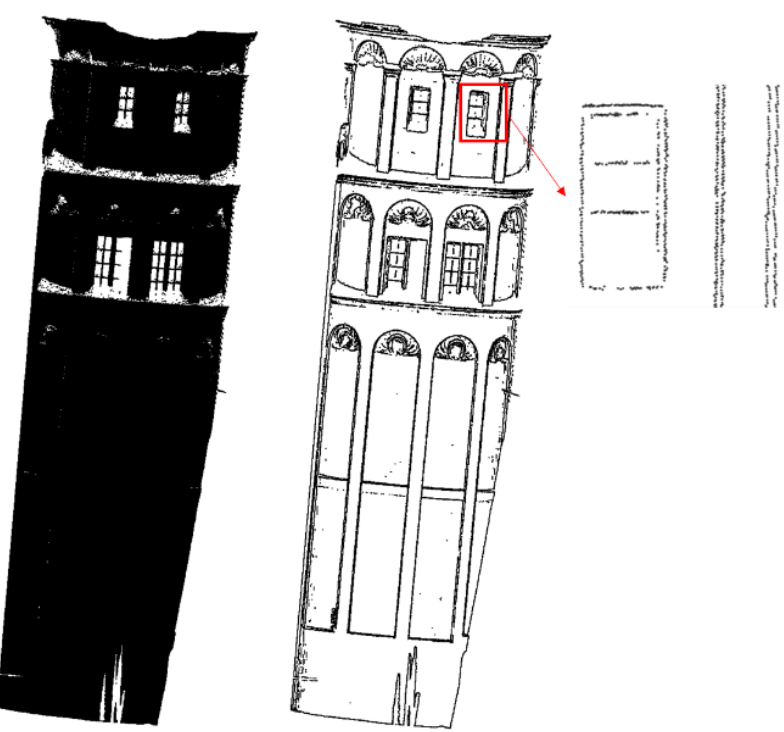

Figure 1. The 3D-edge detection result of a curve building facade, the left picture is the input point cloud, the middle picture is the 3D-edges detected by the employed AGPN method, and right picture is a small area of the middle one. 


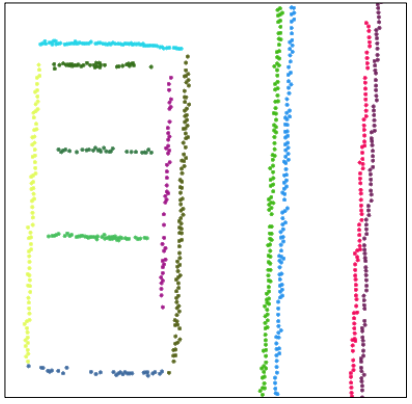

(a)

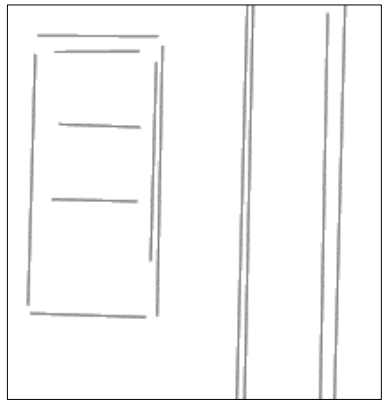

(b)
Figure 2. The result of the feature line tracing procedure in AGPN, (a) is the extracted feature lines, different colours indicate different feature lines, (b) is the extracted straight line segments.

\section{APPLICATIONS OF ALS POINT CLOUD DATA}

In this part, we focus on three applications of ALS point cloud data, i.e., straight line segment extraction, ALS point cloud filtering, and ground breakline extraction.

\subsection{Straight line segment extraction}

To extract 3D straight line segment, we borrow the idea from 2D image processing. For the commonly used straight line extraction method in 2D image, edges are first extracted from the image. Then, a further step of edge linking is performed to determine which contour the edge belong to. At last, straight line segment is extracted from the linked edges, and its line model coefficients are computed. The AGPN method which detects two types of 3Dedges lays the foundation of this procedure in a 3D case. We first employ AGPN method to detect edges in this paper, next, amend the feature line tracing procedure to extract straight lines. Finally, 3D line model coefficients will be computed, and endpoints will be determined to depict a 3D straight line segment. This procedure is straightforward, however, have not been studied. The flowchart of this procedure is shown in figure 3.

Particularly, the feature line tracing step in AGPN method should be amended to extract straight line segment. The reason is that the feature line tracing step in AGPN is designed for smooth curves rather than straight lines. To extract straight feature lines, the step c in Section 2 should be changed, i.e., the comparison of principle directions between the current seed and current neighbours should be replaced by the comparison between the initial seed and the current neighbors.

After straight feature lines are traced, the coefficients of 3D-line model constructed by the points in the same feature line are computed. Let $p_{i}=\left(x_{i}, y_{i}, z_{i}\right)$ for $i=1,2, \ldots, N$, be the points in each feature line. First, the covariance matrix of these points are estimated according to the following formula:

$$
M=\frac{1}{N-1} \sum_{i=1}^{N}\left(p_{i}-p_{u}\right)\left(p_{i}-p_{u}\right)^{T},
$$

where $p_{u}$ is the mean vector of all the points in the feature line. Next, the eigenvectors and eigenvalues are computed. Then the eigenvector $\vec{d}=(a, b, c)$ according to the largest eigenvalue is the principle direction of the 3D-line. The equation of the 3D-line model is:

$$
\frac{x-x_{p u}}{a}=\frac{y-y_{p u}}{b}=\frac{z-z_{p u}}{c},
$$

To depict the 3D-line model, the endpoints of a straight line segment is determined. First, all the points $p_{i}=\left(x_{i}, y_{i}, z_{i}\right)$ for $i=1,2, \ldots, N$ are projected onto the computed 3D-line model. Then, the minimum and maximum extents of these projected points are considered as the two endpoints of a straight line segment.

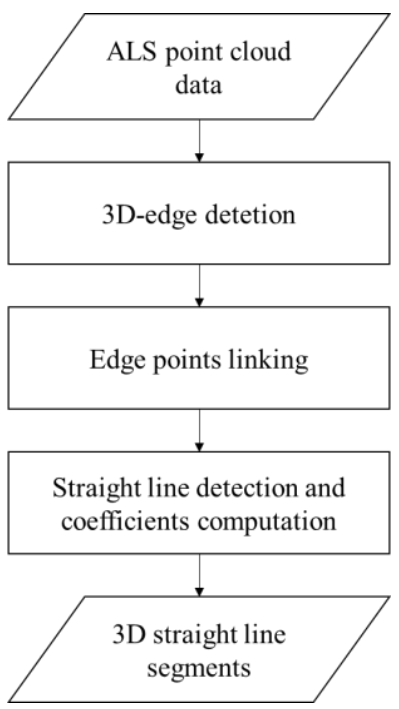

Figure 3. 3D straight line segment extraction method.

\subsection{ALS point cloud filtering}

This study aims at applying AGPN method to progressivedensification-based filters with an assist of point cloud segmentation. This kind of filtering methods is composed of four steps, i.e., point cloud segmentation, initial ground segment selection, initial TIN construction, and iterative densification. To relieve the computational burden of initial TIN construction, Lin et al. (2016) have proposed a way that feature points are utilized to replace all the points in a surface segment. Then, the initial TIN is constructed by the feature points in the initial ground segments, and each segment is depicted by its feature points in the iterative densification procedure. If the feature points are boundaries without fold edges (Ni et al., 2016) and the segment is in a region composed of smooth surfaces and rugged terrains, these feature points cannot depict the geometric properties of the region. The reason is that the ground breaklines and jump edges in rugged terrains are missed in these feature points.

AGPN method which detects boundary elements and fold edges is able to deal with the rugged terrains in a region, hence the $3 \mathrm{D}$ edges detected by AGPN are utilized to replace the commonly used boundaries of surfaces. The flowchart of the improved ALS point cloud filtering procedure is shown in figure 4 .

where $\left(x_{p u}, y_{p u}, z_{p u}\right)$ is the coordinates of $p_{u}$. 


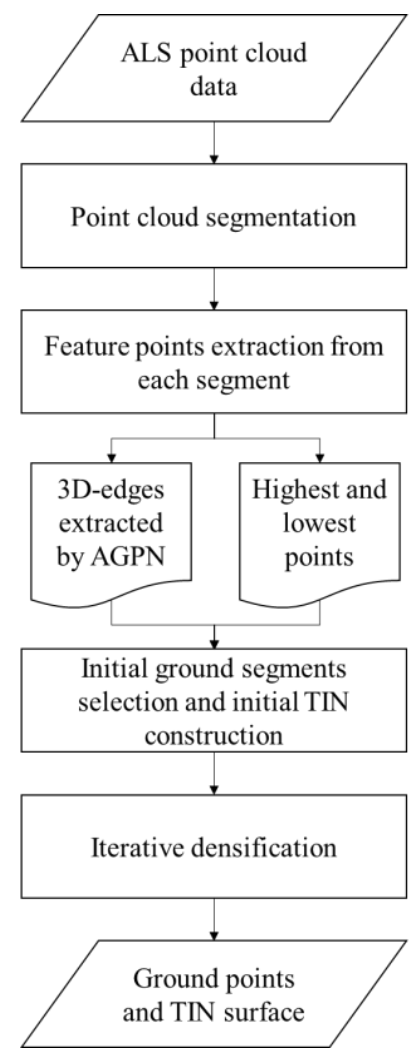

Figure 4. The improved ALS point cloud filtering method.

\subsection{Ground breakline extraction}

According to the aforementioned review of the ground breakline extraction methods for ALS point clouds, current methods often convert ground points into range images. The latest approach (Yang et al., 2016) utilizes this idea and achieves an excellent performance. However, handling ALS point clouds with 3D geometric information by converting to $2 \mathrm{D}$ image with relatively poor spatial information is confused. According to the definition of ground breaklines (Brugelmann, 2000), the ground breaklines include three types, i.e., jump edges, crease edges, and curvature edges. The three types of ground breaklines are shown in figure 5 . These types of breaklines are able to be detected by AGPN according to the definition of the 3D edges in Ni et al., (2016). AGPN method gives an alternative way to extract ground breaklines directly without converting $3 \mathrm{D}$ point clouds into range images.

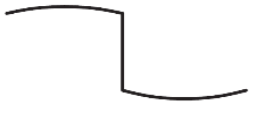

Jump Edge

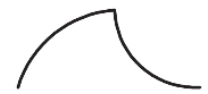

Crease Edge

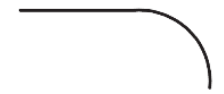

Curvature Edge
Figure 5. Three types of ground breaklines defined in Brugelmann, (2000).

The ground breakline extraction method only includes three steps. First, ground points are extracted by an ALS point cloud filtering method. In this paper, we use Axelsson's filtering method (Axelsson, 2000). Then, 3D-edges are detected by AGPN. At last, the detected 3D-edges are linked to breaklines.
It is notable that AGPN is also utilized in the aforementioned filtering method. The two utilizations of AGPN are different. In the aforementioned filtering method, the AGPN is utilized to extract 3D-edge points for each segment, while it is employed to perform the ground points in the ground breakline extraction.

\section{EXPERIMENTS RESULTS AND DISCUSSION}

To validate the ideas in this paper, we implement all the related methods for ALS point clouds data using $\mathrm{C}++$ language and the Point Cloud Library (PCL). The experiments are conducted on a workstation running Microsoft Windows $7(\times 64)$ with two 16Core Intel Xeon E5-2650, 64GB Random Access Memory (RAM) and 3TB hard disk.

\subsection{Testing data}

Two datasets are involved in our experiments. The first one is a publicly available ALS dataset which is obtained by the University of Iowa in 2008. The data are collected to survey the Iowa River Flood along the Iowa River and Clear Creek Watershed. The data collection is funded by NSF Small Grant for Exploratory Research (SGER) program. The dataset is able to be accessed in OpenTopology. An urban area is selected, and is utilized to test the performance of straight line segment extraction. The buildings in the urban area has complex roof structures which are depict by the ALS point clouds in detail. The average point spacing of the testing area is $0.6 \mathrm{~m}$. In contrast with groundbased laser scanning point cloud data, ALS point cloud data presents more building roofs rather than building facades. Therefore, some methods designed for ground-based laser scanning point cloud data are not suitable for ALS point cloud data. The original paper ( $\mathrm{Ni}$ et al., 2016) which proposes the AGPN method tests the performance using dense point cloud obtained by ground-based laser scanning system. The performance of AGPN method for ALS point cloud with relatively sparse points and large point spacing is unknown. Therefore, the area of ALS point cloud data is not only utilized to test the straight line segment extraction, but also utilized to test the performance of AGNP method for ALS point cloud data. The area is shown in figure 6 , and coloured by elevation.

The second one is the ISPRS testing dataset for ALS point cloud filtering, which is published by ISPRS Commission III, Working Group III. The dataset is obtained by an Optech ALTM scanner over the Vaihingen/Enz test field and the Stuttgart city center. It includes eight sites consisting different terrains: four urban sites and four rural/wooded sites. To facilitate the testing of different filtering methods, the dataset also provides 15 reference samples of sub-areas. The characteristics of the dataset are shown in Sithole and Vosselman (2004). The dataset is utilized to test the performance of the ALS point cloud filtering and ground breakline extraction.

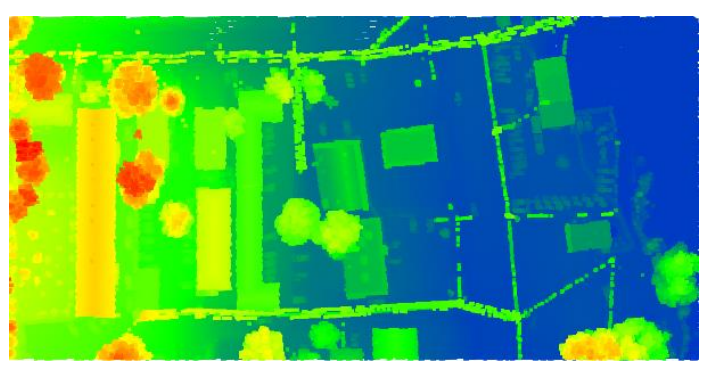

Figure 6. The testing area for 3D straight line segment extraction. 


\subsection{Parameter setting}

There are two parameters should be determined when we use AGPN to detect 3D-edges, i.e., the distance threshold $d_{r}^{1}$ for RANSAC plane model estimation, and the number of nearest neighbors $K^{1}$. In the original paper (Ni et al., 2016) of AGPN, the result is sensitive to $d_{r}^{1}$. Its experimental analysis shows that good results are able to be obtained when $d_{r}^{1}$ is equal to the mean point spacing of ground-based laser scanning point cloud data. However, when AGPN deal with coarser ALS point clouds with large point spacing, setting $d_{r}^{1}$ to mean point spacing is unsuitable. Herein, we set $d_{r}^{1}$ to 0.1 empirically. The reason is that we should ensure the precision of local RANSAC plane model estimation. Moreover, $K^{1}$ should be set to a small value in contrast to the setting of ground-based laser scanning point cloud data. Herein, we set $K^{1}$ to 50 empirically.

In the applications of straight line segment extraction, and ground breakline extraction, the feature line tracing step of AGPN is used. There are three parameters in this step, i.e., the number of nearest neighbors $K^{2}$, the distance threshold $d_{r}^{2}$ for the RANSAC line model estimation, and the smooth direction threshold sm_thr. According to the aforementioned analysis and experiments, we set $K^{2}=15, d_{r}^{2}=0.1$, and $s m_{t h r}=0.1$ empirically.

\subsection{D Straight Line Segment Extraction}

The testing area in the first dataset is performed by the 3D straight line segment extraction procedure presented in Section 3.1. The results are presented in figure 7.

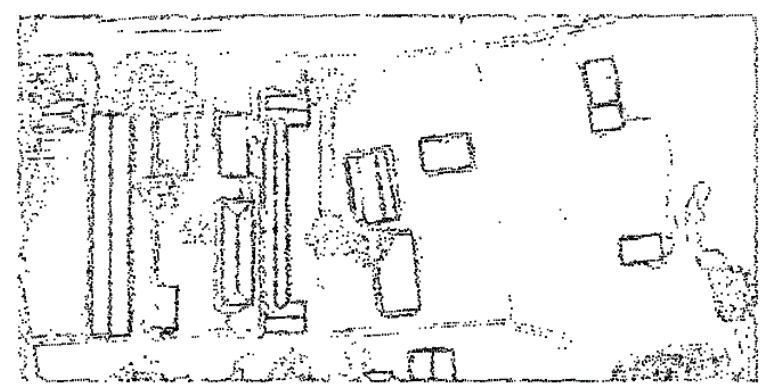

(a)

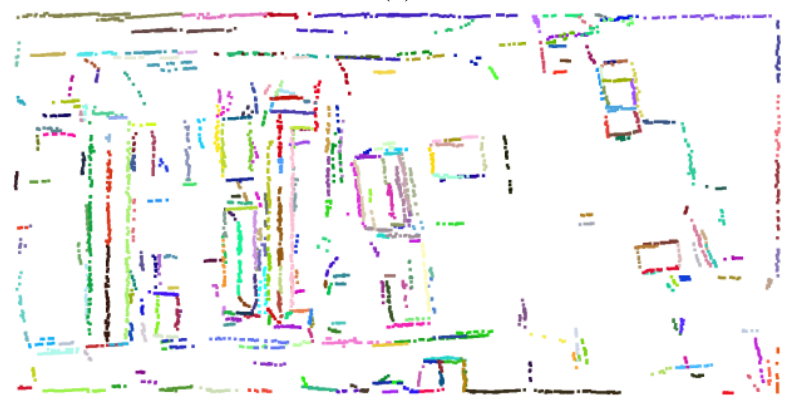

(b)

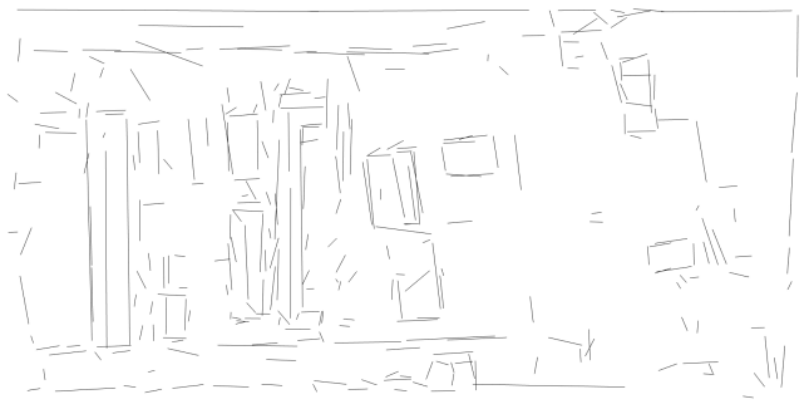

Figure 7. The results of straight line segment extraction for the testing area in the first dataset, (a) is the 3D-edges detected by AGPN, (b) is the traced feature lines, (c) is the extracted straight line segments.

From the results presented in Figure 7, the performance of 3Dedge detection and feature line tracing for ALS point clouds is worse than that for ground-based laser scanning point cloud data. Only $47.3 \%$ straight line segments are correctly extracted, however, when the method is performed on a ground-based laser scanning data with average point spacing $0.01 \mathrm{~m}$, the rate is able to reach $80 \%$. The reason is that the point spacing of ALS point clouds is larger than that of ground-based laser scanning point cloud. However, nearly all the 3D-edges including boundary elements and fold edges are detected. The most serious problem of the straight line segment extraction is that long straight line segments are more likely to be divided into several short ones. If we use a dense point cloud with smaller point spacing such as $0.01 \mathrm{~m}$, the problem will be solved.

\subsection{ALS Point Cloud Filtering}

In general, boundaries except for fold edges are utilized to represent the geometric properties of a ground surface, and then an initial TIN is constructed using these boundaries, and the highest and lowest points. These feature points in the initial ground surface are shown in figure $8 \mathrm{~b}$. We can find that the feature points depict a ground surface properly when the ground surface is flat. However, when the ground surface is rugged with steps, these feature points will fail to depict it. In contrast with boundaries, the 3D-edges detected by AGPN is able to overcome this problem and include all the edges of the rugged terrains (see figure $8 \mathrm{c}$ ). Therefore, we utilize 3D-edges, the highest and lowest points of a surface segment to replace the aforementioned feature points. To quantitatively analyse the performance of this replacement, we test the procedure on the 15 reference samples of the second dataset. The mean, maximum and minimum TYPE I, TYPE II and TOTAL errors are computed and shown in table 1. To simplify the expression, the points including boundaries, the highest and lowest points are called feature points. The filter using these feature points is the original method, and the filter using 3D-edges detected by AGPN is our improved method. According to table 1, although the areas of the 15 samples are too small to show a large extent of error reduction, all the errors of our improved method are lower than the original method. This demonstrates the benefits of the utilization of AGPN.

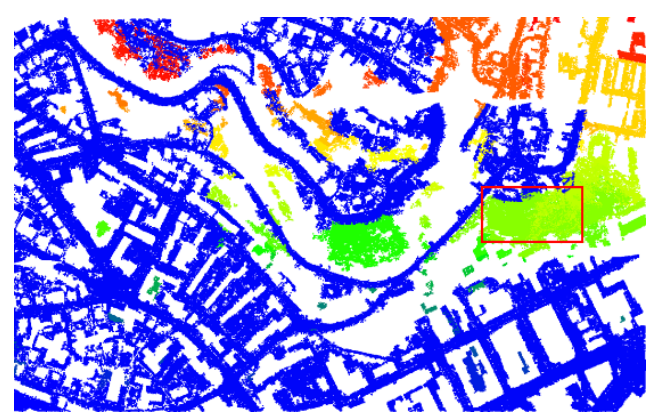

(a)

(c) 


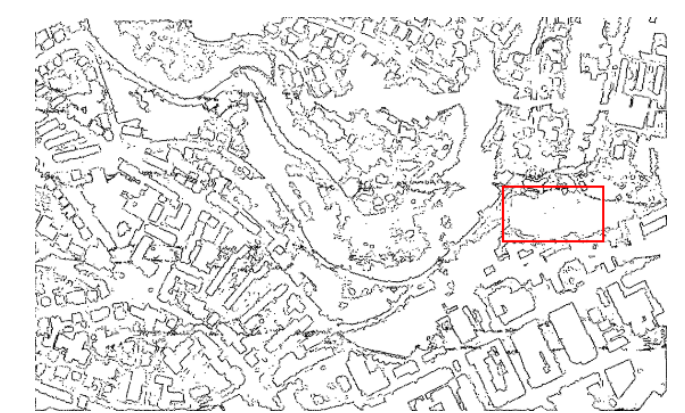

(b)

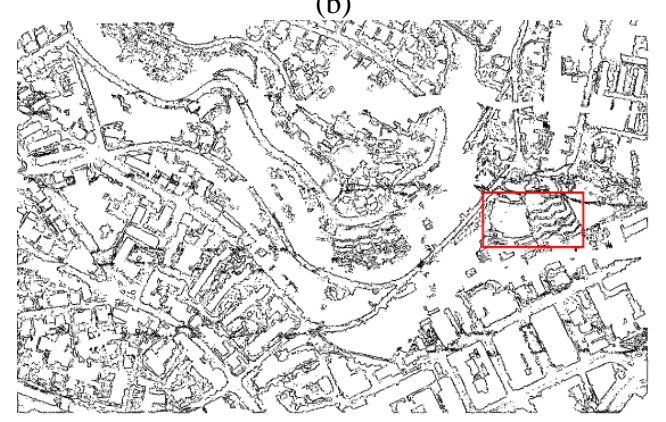

(c)

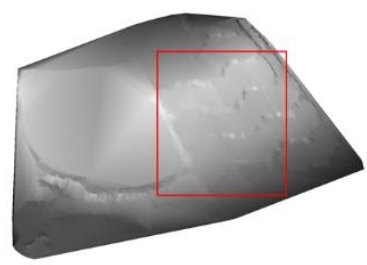

(d)

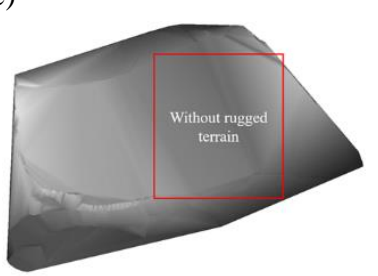

(e)

Figure 8. Results of the initial ground surface construction by TIN for the Cite1 in the second dataset, (a) is the selected initial ground surfaces (depicted by segments), (b) is the feature points including boundaries, the highest and lowest points, (c) is the 3D-edges detected by AGPN method, (d) is the TIN surfaces constructed by $3 \mathrm{D}$-edges detected by AGPN, the small area corresponds to the red rectangles in (a)-(c), (e) is the TIN surfaces constructed by feature points, it corresponds to the area in (d).

\begin{tabular}{|l|l|l|l|l|l|l|}
\hline $\begin{array}{l}\text { Metho } \\
\text { ds }\end{array}$ & \multicolumn{3}{|c|}{ Our improved method } & \multicolumn{3}{c|}{ The original method } \\
\hline $\begin{array}{l}\text { Types } \\
\text { of } \\
\text { errors }\end{array}$ & $\begin{array}{l}\text { TYP } \\
\text { E I I }\end{array}$ & $\begin{array}{l}\text { TYP } \\
\text { E II } \\
(\%)\end{array}$ & $\begin{array}{l}\text { TOT } \\
\text { AL } \\
(\%)\end{array}$ & $\begin{array}{l}\text { TYP } \\
\text { E I } \\
(\%)\end{array}$ & $\begin{array}{l}\text { TYP } \\
\text { E II } \\
(\%)\end{array}$ & $\begin{array}{l}\text { TOT } \\
(\%)\end{array}$ \\
\hline max & 27.0 & 42.5 & 27.8 & 28.2 & 43.6 & 28.0 \\
min & 0.3 & 2.4 & 3.2 & 0.3 & 2.4 & 3.4 \\
mean & 6.8 & 20.0 & 9.4 & 6.8 & 20.8 & 9.5 \\
\hline
\end{tabular}

Table 1. Comparison between the two filters using feature points and 3D-edges.

\subsection{Ground breakline extraction}

After the ground surfaces are extracted by an ALS point cloud filtering method, the ground breaklines will be determined by performing AGPN method on the ground surfaces. The breaklines extracted by AGPN is shown in figure 9 .
From figure 9, there are some errors in the extraction result. The performance of the ground breakline extraction can hardly surpass the method in Yang et al., (2016). A close-up visual inspection shows that the testing data has a low point density. The low density results in the problem that a long breakline is divided into several short ones, which is the most serious problem of the ground breakline extraction in this paper.

The method for extracting ground breaklines in this paper is more sensitive to point densities than the method in Yang et al., (2016). However, we present a novel and straightforward way for this application without converting $3 \mathrm{D}$ point clouds into range images.

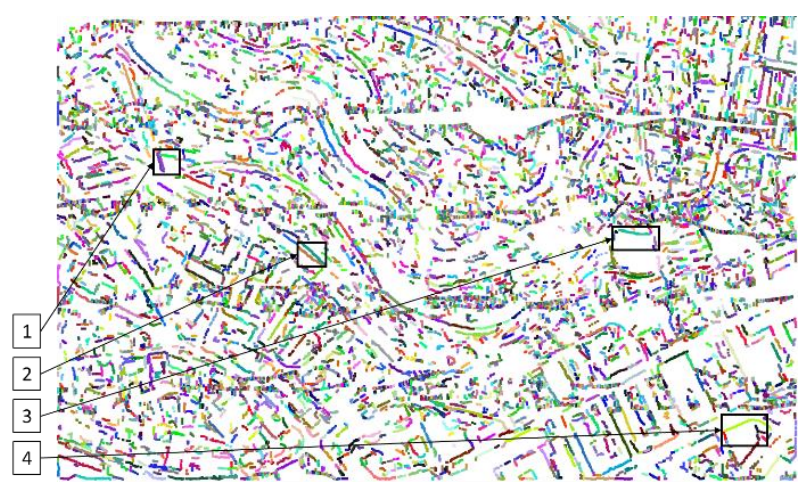

Figure 9. Breaklines extracted by AGPN of the Cite1 in the second dataset.
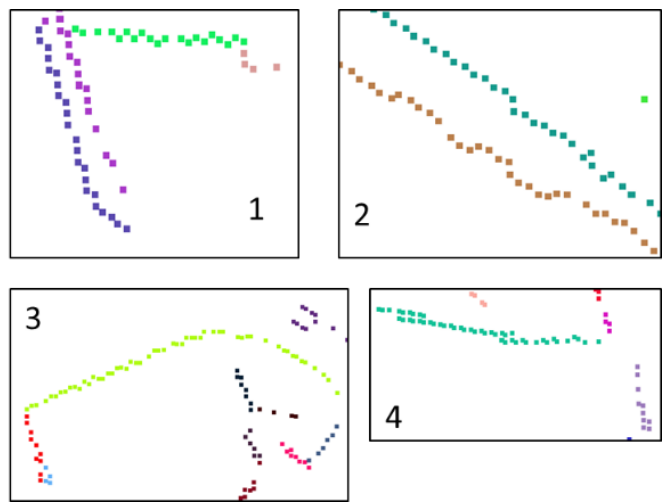

Figure 10. The results of the four small areas corresponding to figure 9 .

\section{CONCLUSION}

In this paper, we present three applications of AGPN method for ALS point cloud data. The AGPN method is utilized to extract 3D straight line segments, filter ALS point cloud, and extract ground breaklines. However, it needs high point density of ALS point cloud data if good results of all three applications are obtained.

In the near future, with the improvement of the airborne laser scanning system, the point density of ALS point clouds will be further improved, and acquiring an ALS point cloud as dense as ground-based laser scanning data may be realizable. In this case, the AGPN method will have more and more significant applications.

\section{ACKNOWLEDGEMENTS}

This research was funded by: (1) the General Program sponsored by the National Natural Science Foundations of China (NSFC) 
under Grant 41371405 and 41671440; (2) the Foundation for Remote Sensing Young Talents by the National Remote Sensing Center of China.

\section{REFERENCES}

Axelsson, P.E, 2000. DEM generation from laser scanner data using adaptive TIN models. International Archives of the Photogrammetry, Remote Sensing and Spatial Information Sciences, 32, pp. 110-117.

Borges, P., Zlot, R., Bosse, M., Nuske, S., Tews, A, 2010. Visionbased localization using an edge map extracted from 3D laser range data. In: Proceedings of the International Conference on Robotics and Automation (ICRA), Anchorage, AK, USA, pp. 37.

Brügelmann, R., 2000. Automatic breakline detection from airborne laser range data. International Archives of the Photogrammetry, Remote Sensing and Spatial Information Sciences, 33, pp. 109-116.

Demarsin, K., Vanderstraeten, D., Volodine, T., Roose, D, 2007. Detection of closed sharp edges in point clouds using normal estimation and graph theory. Computer-Aided Design, 39(4), pp. 276-283.

Gumhold, S., Wang, X., Macleod, R, 2001. Feature extraction from point clouds. In: Proceedings of the 10th International Meshing Roundtable, Sandia National Laboratory, Newport Beach, CA, USA, pp. 7-10.

Li, H., Zhong, C., Hu, X.G., 2013. New methodologies for precise building boundary extraction from LiDAR data and high resolution image. Sensor Review, 2, pp. 157-165.

Lin, Y.B., Wang, C., Cheng, J., 2015. Line segment extraction for large scale unorganized point clouds. ISPRS Journal of Photogrammetry and Remote Sensing, 102, pp. 172-183.

Lin, X., Zhang, J., 2014. Segmentation-based filtering of airborne lidar point clouds by progressive densification of terrain segments. Remote Sensing, 6, pp. 1294-1326.

Ni, H., Lin, X., Ning, X., Zhang, J., 2016. Edge detection and feature line tracing in 3D-point clouds by analyzing geometric properties of neighborhoods. Remote Sensing, 8, pp. 710-729.

Poullis, C., 2013. A framework for automatic modeling from point cloud data. IEEE Transactions on Pattern Analysis and Machine Intelligence, 11, pp. 2563-2575.
Sampath, A., Shan, J., 2010. Segmentation and reconstruction of polyhedral building roofs from aerial lidar point clouds. IEEE Transactions on Geoscience and Remote Sensing, 48, pp. 15541568.

Sithole, G., Vosselman, G., 2004. Experimental comparison of filter algorithms for bare-earth extraction from airborne laser scanning point clouds $\bar{i}$. ISPRS Journal of Photogrammetry and Remote Sensing, 59, pp. 85-101.

Sohn, G., Dowman, I., 2002. Terrain surface reconstruction by the use of tetrahedron model with the mdl criterion. International Archives of the Photogrammetry, Remote Sensing and Spatial Information Sciences, 34, pp. 336-344.

Truong-Hong, L., Laefer, D.F., Hinks, T., 2013. Combining an angle criterion with voxelization and the flying voxel method in reconstructing building models from LiDAR data. ComputerAided Civil and Infrastructure Engineering, 28(2), pp. 112-129.

Von Gioi, R.G., Jakubowicz, J., Morel, J.-M., Randall, G., 2010. Lsd: A fast line segment detector with a false detection control. IEEE Transactions on Pattern Analysis and Machine Intelligence, 32, pp. 722-732.

Yang, B., Huang, R., Dong, Z., Zang, Y., Li, J., 2016. Two-step adaptive extraction method for ground points and breaklines from lidar point clouds. ISPRS Journal of Photogrammetry and Remote Sensing, 119, pp. 373-389.

Zhang, J., Lin, X., 2013. Filtering airborne lidar data by embedding smoothness-constrained segmentation in progressive tin densification. ISPRS Journal of Photogrammetry and Remote Sensing, 81, pp. 44-59.

Zhang, J., Lin, X., 2017. Advances in fusion of optical imagery and lidar point cloud applied to photogrammetry and remote sensing. International Journal of Image and Data Fusion, 8, pp. $1-31$.

Zhang, K., Chen, S., Whitman, D., Mei-Ling, S., Yan, J., Zhang, C., 2003. A progressive morphological filter for removing nonground measurements from airborne lidar data. IEEE Transactions on Geoscience and Remote Sensing, 41, pp. 872882.

Revised June 2017 